\title{
Unlocking Potentials of Building Energy Systems' Operational Efficiency: Application of Digital Twin Design for HVAC systems
}

\author{
Christian Vering ${ }^{1}$, Philipp Mehrfeld ${ }^{1}$, Markus Nürenberg ${ }^{1}$, Daniel Coakley ${ }^{2}$, \\ Moritz Lauster ${ }^{1}$, Dirk Müller ${ }^{1}$ \\ ${ }^{1}$ Institute for Energy Efficient Buildings and Indoor Climate, Aachen, Germany \\ ${ }^{2}$ Mitsubishi Electric, Edinburgh, Scotland
}

\begin{abstract}
Building energy systems are complex because of their nonlinear behaviour and stochastic environmental interactions. Thus, their efficiency depends on both component design and control strategy. Aiming for high efficiencies over the whole lifecycle, we apply Product Lifecycle Management and Digital Twin Design to HVAC systems, initially using an energy recovery ventilation (ERV) simulation model.

We develop a Digital Twin Prototype in Modelica of the ERV unit to predict physical system behaviour. Verifying functionality and suitability, we set up a Digital Twin Instance (DTI) by calibrating the model against measurement data of the physical twin. DTI and physical twin communicate via MQTT interfaces with each other.

The approach enables DTIs to calculate predictions for different scenarios per instance, which increases systems' efficiency significantly. In conclusion, this framework allows an application of innovative and predictive control and maintenance strategies to HVAC systems.
\end{abstract}

\section{Introduction}

Digitalization of energy systems enables the application of sophisticated control strategies increasing operational efficiencies significantly (Afram, 2014). Unlocking the entire potential, there is an urgent need to design systems carefully by simultaneous consideration of functional requirements and control strategies with digital interfaces in early development stages (Dongellini 2017; Poppi, 2016). However, integral descriptions that face these concepts for HVAC systems do not exist, yet. On the one hand, an integral product development approach regarding the whole lifecycle is required. We propose Product Lifecycle Management to be suitable (Stark, 2015). On the other hand, the digital twin approach has proven great suitability for complex system designs and their operation (Grieves, 2017). A combination of both concepts for HVAC Systems has currently not been found in literature.

Building energy systems are complex because of their nonlinear behaviour and stochastic environmental interactions (Staffel, 2012). Furthermore, HVAC systems make up the majority of commercial building energy consumption, and are directly responsible for maintaining the health and wellbeing of occupants in Germany (Arbeitsgemeinschaft Energiebilanzen e.V., 2017). However, their efficiency strongly depends on both component design and control strategy (Afram, 2014). Aiming for high efficiencies, we apply systematically Digital Twin Design (DTD) to HVAC systems, initially using a detailed model of an energy recovery ventilation (ERV) unit.

Thereby, this work contributes to the application of the digital twin approach to HVAC systems by example of an energy recovery ventilation unit considering Product Lifecycle Management:

- We combine the approaches of Product Lifecycle Management and Digital Twin Design (DTD) to demonstrate its potential for the use in realistic, manufacturing applications in Chapter 2.

- In Chapter 3, we show the modelling approach of the ERV unit in Modelica and a room model to apply it to a use case. We calibrate the ERV model using measurement data.

- A use case of predictive maintenance is evaluated in Chapter 4 were we evaluate different energy consumptions due to different maintenance strategies.

- Finally, we summarize and conclude our findings.

\section{Digital Twins in Product Lifecycle Management}

Product Lifecycle Management (PLM) is a production engineering approach for the holistic control and administration of product-related information along the entire products' lifecycle. Methods, models and tools (especially IT systems) support the product development process and the product usage phase in order to increase productivity and efficiency. PLM integrates all data and information collected along the product lifecycle. (Stark, 2015)

The first use of the term in literature dates back to the beginning of the 21 st century. A uniform definition of the exact components that a PLM system must present in order to be declared as such does not exist. Previous applications refer mainly to physical products and processes (Tao, 2018). In the context of this work, PLM is to be additionally implemented for virtual models in the context of digital twins. (Stark, 2015)

PLM distinguishes between fife phases of products' lifecycle. In the planning phase, a product is defined on the basis of customer requirements, market analyses, innovative ideas and knowledge from former products. A preliminary design is carried out in which technical 
parameters and functions are defined. These are concretized in the create-phase by first models or prototypes. In the build phase, production is finally started based on the defined product design. The following use of the product is described in the sustain phase. This also includes services such as maintenance and repair. Ultimately, the disposal phase after the end of use includes processes for disposal or recycling. (Yoga Mule, 2012)

At the moment, there is no standardized definition of what exactly a digital twin is. A widespread understanding describes the term digital twin as an intelligent, digital image of a real product or process. Against this background, a separate approach to the implementation of a digital twin will be developed within the presented framework. The first approaches to the implementation of a digital twin come from the field of aeronautics and space technology. In this context, NASA in particular should be mentioned as a "pioneer" in the development and use of digital twins. Their intention to use digital twins is to monitor the functional condition of an aircraft or space shuttles as an overall system as well as individual components. (Schleich 2017; Tuegel, 2011)

NASA defines a digital twin e.g. as follows: "A Digital Twin is an integrated multiphysical simulation of a vehicle or system actually built, using the best available physical models, sensor technologies, fleet history, etc., to reflect the life of the corresponding flying twin (Schleich, 2017). Grieves, who worked in an advisory capacity for NASA, is considered the founder of the term digital twin, which he describes in the context of PLM (Grieves, 2014). It defines a digital twin as "a set of virtual information that completely describes a potential or actually manufactured physical product from the micro atomic plane to the macro geometric plane". (Grieves, 2017)

Combining both approaches, PLM and digital twins, and applying them to HVAC systems, we propose Digital Twin Design.

Digital Twin Design has PLMs' fife phases. These phases are combined with digital twin definitions. Regarding (Grieves, 2017), we combine three PLM phases with existing digital twin definitions:

\section{Create phase $\rightarrow$ Digital Twin Prototype (DTP) \\ Build phase $\rightarrow$ Digital Twin Instance (DTI) \\ Sustain phase $\rightarrow$ Digital Twin Aggregate (DTA)}

In addition, we propose two further Digital Twins to be on PLM phases:

\section{Dispose phase $\rightarrow$ Digital Twin Knowledge (DTK) \\ Plan phase $\rightarrow$ Digital Twin Concept (DTC)}

The first step in product development is the creation of a Digital Twin Concept (DTC). The conception takes place in the PLM plan phase. It comprises the entire planning and design of a concept for the implementation of a digital twin for HVAC systems. Based on customer needs, market analyses and individual ideas, goals are derived which are pursued by the implementation of a digital twin.
Once these objectives have been defined, a requirements analysis can be carried out to define product characteristics, functions and operating strategies. Besides former typical requirements such as physical concepts, in this phase requirements for the virtual model need to be set up.

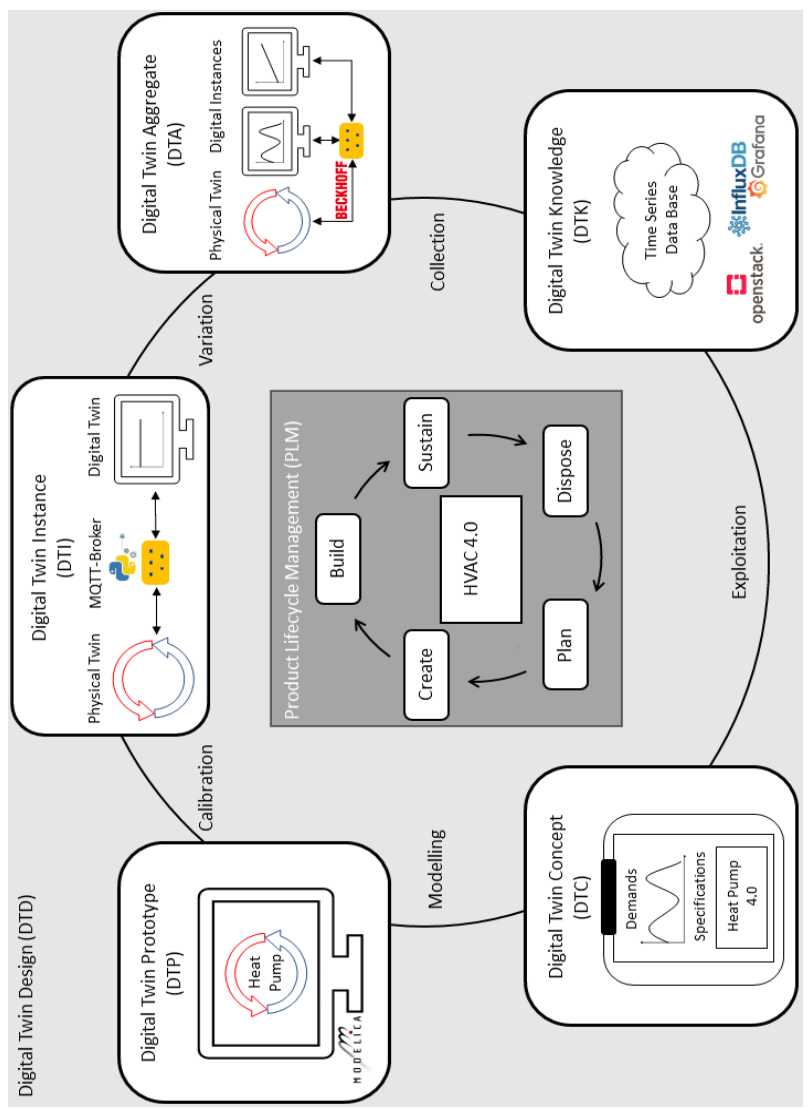

Figure 1: Applying Product Lifecycle Management and digital twins to Digital Twin Design for HVAC systems.

The first requirement of the virtual model is the definition of model's inputs and outputs. Applied to HVAC systems, typical inputs are measurement data points like volume flows, temperatures and pressure levels. Based on this inputs our model needs to predict outputs. Typically these are power consumptions e.g. of fans, pumps or heaters.

If inputs and outputs are defined, a second requirement needs to be met. Regarding the application it has to be defined, which level of accuracy the model should has. In general, we can distinguish between

- black box,

- grey box and

- white box model.

Black box models are mathematical models, whereas grey box models use physical equations and some simplifying assumptions to represent physical system behaviour. White box models are a perfect description of the physical system. With an increasing level of detail, calculation durations of model predictions increase as well. Consequently, the trade-off between accuracy and simulation speed needs to be clarified in an early design stage. (Mueller, 2016) 
Creating the Digital Twin Prototype (DTP) in the PLM create phase, a simulation model of the HVAC system must be created. In addition to modelling, the subsequent parameterization of the model is also carried out in the DTP phase. In our use case of an ERV system, we need simulation models of heat exchangers, ventilators, air filters, heater, cooler, temperature control, sources, sinks and the target room. Therefore, we use Modelica because of both modelling of dynamic systems as well as a high degree of modularity and reusability.

After modelling all components, a parametrisations needs to be done. For this purpose, safe, known parameters are transferred into the model. In particular, geometric boundary conditions must be taken into account, such as heat-transferring surfaces, wall thicknesses, room dimensions, etc. The materials used for the individual components are also among the parameters known in many applications.

Assumptions can be made for parameters that are not known or are subject to uncertainties. However, it must be borne in mind that this creates uncertainties in the overall system, which can lead to a deviation between simulation and measured values. Due to uncertain parameters, a subsequent calibration is of enormous importance when modelling real systems. With the completion of the simulation model, the prototype of the digital twin exists. The mentioned calibration of the simulation model is intended as a transition to the build phase according to DTD.

The creation of a Digital Twin Instance (DTI) in the PLM build phase requires a calibrated simulation model and a communication structure between physical and digital twins. The calibration of the simulation model serves to map selected output variables of the real system through the simulation. Any deviations between measured values and simulation results should be as small as defined in the planning phase.

Several methods exist for the calibration of HVAC systems. Bayesian calibration has proven to be suitable for dynamic systems. This is mainly due to the good predictability of output variables, so that there is a high probability that measured values will be well represented by the simulation after calibration (Li, 2017).

The disadvantages of the high complexity and numerous required simulations are accepted. There is a promising approach in the literature for the implementation of Bayesian calibration (Chong, 2018):

1) Generate/procure measurement data

2) Sensitivity analysis/parameter testing

3) Generate simulation results

4) Combining measurement data and simulation results in Gaussian process

5) Distribution determination (Markov Chain Monte Carlo method)

6) Check convergence and assess the accuracy of the calibrated model.
In the course of the development of the DTD approach, a (partially) automated implementation of this methodology for digital twins is considered to be promising. A practical implementation for the simulation model of the ventilation system has not yet taken place. The existing data basis was not sufficient in this respect. Instead, a manual calibration of the model is carried out.

The target values of the model and therefore for calibration are power consumption of the fan $\left(P_{\mathrm{el}}\right)$ on the one hand and the effectiveness of the heat transfer in the cross-flow heat exchanger $\left(\varepsilon_{\text {Cross }}\right)$ on the other hand. Measured values from the manufacturer were available in this respect. For several operating points, different volume flows per stage and related power consumptions are available. The calibration was conducted fitting the heat transition coefficient of supply air, heat exchanger material and exhaust air in order to match the both target values.

The Root Mean Square Error (RMSE) is used as the criterion for assessing the quality of the calibration. The RMSE is calculated as follows (Mehdiyev, 2016):

$$
R M S E=\sqrt{\frac{\sum\left(y_{\text {sim }}-y_{\text {Meas }}\right)^{2}}{n}} .
$$

First, $n$ simulation results $y_{\text {sim }}$ are generated with the non-calibrated model. By comparison with measured $y_{\text {Meas }}$ values, a deviation for the target values can be determined. The lower the RMSE the better a calibration was conducted. With this calibrated model, Digital Twin Design is ready to use.

In order to exploit the benefit of a Digital Twin Design, digital and physical twins are constantly exchanging data. Ensuring this requirement, an infrastructure is required on which communication can take place. In the following, we will show how such a communication interface can be implemented to enable data transfer between digital and physical instances as well as data storage in a cloud. Based on the results of extensive literature research, MQTT (Message Queuing Telemetry Transport) has proven to be suitable for implementing communication structures for IoT applications. (HiveMQ, 2019)

Notably, low demands on the digital infrastructure, such as network and bandwidth, make the almost universal use of the digital twin possible. In addition, an enormous number of end devices can participate in this communication structure. The intention of executing several instances of the digital twin in parallel, MQTT has many advantages. Hirmer et al. (Hirmer, 2016) present an approach for the implementation of an architecture for automated communication for IoT environments. The easily scalable communication protocol MQTT is used. Hence, the access to sensor data of physical systems is realized. Communication with digital instances takes place on a publish/subscribe architecture. Haag and Anderl (Haag, 2018) describe the implementation of a digital twin based on an MQTT architecture.

In addition to the communication between the physical and digital instance, a link is provided to display data and results in real time via a graphical user interface. Based 
on this implementation, potentials with regard to monitoring and the resulting ease of use for users can be derived.

The MQTT-Broker as central instance for communication manages the data transfer between physical and digital instances as well as the storage of data in a cloud. All subscribers (terminals) send requests to the MQTT broker using the commands publish and subscribe, which ensures their implementation. Data is made available by publish, while data is requested by subscribe.

Meeting requirements of communication services, the Quality of Service (QoS) level should be at least 1, as this ensures that data arrives. In order to ensure that the data arrive exactly once, QoS level 2 must be selected, which however results in increased data traffic due to increased communication. If the resource requirement plays a minor role due to increased data traffic, this disadvantage can be ignored and level 2 can be implemented. (HiveQM, 2019)

With regard to communication with the physical instance, an approach to implementation has been developed as part of further work. A transfer of the developed approach to the DTD is planned, but must still be tested in practice. To implement communication on the part of the digital instances, an MQTT-Dymola interface is currently under development at the Institute for Energy Efficient Buildings and Indoor Climate (EBC) at RWTH Aachen University. This is to serve as an interface to read simulation results from Dymola (Modelica) and to introduce parameters into Dymola.

The implementation of the communication interface completes the build phase. A functional instance of the Digital Twin (DTI) is available. In the following, the developed DTD approach provides for the aggregation and execution of several instances the sustain phase.

The simultaneous execution of several DTIs is necessary to achieve some of the objectives of the DTC. In the PLM sustain phase several DTIs are aggregated to Digital Twin Aggregate (DTA). To enable simultaneous, stable execution of the aggregates, a suitable software environment is required. In this respect, known approaches were examined within the scope of this work and a theoretical implementation possibility was integrated into the DTD approach developed.

Virtual machines are a well-known advocate for creating an environment in which software can be run in isolation. Due to some disadvantages of virtual machines, especially in the areas of efficiency and performance, alternatives have gained in importance in recent years. An example of this is Docker (Bauer, 2018). Docker uses containers to create an environment in which software can be run in isolation. Hence, Docker is suitable for the implementation of the DTD approach in the DTA phase due to simple possibilities for instantiating processes.

Individual instances in the Docker containers can simulate different scenarios. These can vary in many ways. Various weather data are just as conceivable as simple parameter variations. These include, for example, a decreasing mass flow due to leakages or additional internal heat gains due to deviating user interaction. This corresponds to carrying out various case studies. In addition, more extensive instances can also be executed, such as complete annual simulations. As mentioned in the description of the DTC phase, the implementation of the DTD approach pursues several objectives like operational optimization or predictive maintenance.

On the one hand, the digital twin can be used to optimize the operation of the physical Twin. Different scenarios of the DTIs are of central importance here. Due to the simulations of the DTIs' carried out parallel to the real operation, simulation results are available for various parameters under the currently prevailing boundary conditions. Based on these results, the digital twin can determine the optimal scenario for the operation and transfer the parameters used to the real system via the developed communication structure. The implementation of the ERV system looks as follows:

Parallel to the operation of the real system, several instances are executed that simulate different scenarios. An instance recreates the current operation of the real system. The other instances vary single or multiple parameters, such as air mass flow or pressure drop due to the use of a new air filter. Different scenarios lead to the fact that the instances deliver different simulation results. Within the framework of operational optimisation, the efficiency of the operation can be determined based on predefined assessment variables (e.g. consumption of electrical energy).

Through the communicated comparison between the individual instances, the most efficient operating point can be determined. The instance that simulated it, transfers the operating parameters to the real system in real time, so that it is executed at the optimum operating point. The required valuation parameters can be very complex in this context. Accordingly, the realization of a digital twin for operational optimization is a complex process. Nevertheless, the idea in connection with resource-saving energy supply in the HVAC sector seems to be promising for the creation of more efficient systems.

Another possible use of the digital twin is predictive maintenance. This is closely related to the ability of the digital twin to monitor the operation of the real system. The digital twin as a virtual image of the real system has the current operating data of the system at its disposal. This makes it possible to monitor the functionality and ensure operation in real time. Based on the evaluation of current operating data, the digital twin can draw predictive attention to maintenance and repair measures. Problems can thus be recognised in advance and ideally solved before they become acute.

By implementing predictive maintenance, maintenance routines can be planned and downtimes are avoided consequently. Both technical and personnel resources can thus be used in an optimised manner. As a result, an increase in the life cycle efficiency of products is achieved. (Schreiner, 2018)

The prerequisite for implementing predictive maintenance is simulation models that provide results in real time and predictively. In addition, the simulation 
models must have the ability to predict malfunctions based on characteristic values. (Schreiner, 2018)

Large amounts of data are generated during the entire sustain phase of the digital twin. Following the DTD, all operating data of the digital twin are stored. After the end of the use in the DTA phase, a data collection is available which is finally used in the last phase of the life cycle.

The last step of the product life cycle (PLM dispose phase) is the disposal of the product. Since the digital twin has processed, generated and stored an amount of data and information in the course of its life cycle, Digital Twin Knowledge (DTK) phase provides evaluation and utilization of the data. In the following, some ideas are shown, how and for which purpose the evaluation of the data generated and stored over the entire life cycle can take place. During the operation of the Digital Twin Aggregate, generated operational data is stored in a cloud via the implemented communication structure. The data thus generated over the entire life cycle can be analysed after active use in the DTK phase. Various software fundamentals are required for the entire data evaluation process. (Meier, 2018)

At its core is an architecture that provides an environment for cloud computing a database that can manage generated time series data from simulations and sensor readings, and a user interface that enables real-time monitoring of operational data. Such an architecture ensures that data generated over the lifecycle of the digital twin can be meaningfully stored, managed and used.

PLM is a closed loop process. After the last phase, the cycle starts again from the beginning. In the DTD, the entire available data basis is consequently transferred to a new life cycle via the evaluation step. This integration takes place in the form of information, experience and knowledge. This step accounts for a large part of the added value of using a digital twin. Based on the transferred information, a second generation of the real product or the corresponding digital twin can be produced, for example. With the help of evaluation as part of the DTK phase, both positive and negative characteristics of the first generation can be evaluated. The aim for the second generation should be to eliminate negative aspects and to strengthen positive aspects.

The DTK phase thus leads to improved products and associated digital twins. To illustrate the DTD, an ERV system will be evaluated using dynamic simulation models, in order to be able to simulate various scenarios for the operation and thus demonstrate the applicability of the DTD. Ultimately, this approach is intended to identify previously hidden potentials with regard to energy and resource efficiency in the life cycle of HVAC systems.

\section{Modelling and Calibration}

Within this work, DTD is applied to an ERV system in order to evaluate its potential of predictive maintenance. Therefore, dynamic simulation models are necessary. These are developed in Modelica and Dymola as compiler. The ERV system is shown in Figure 2.

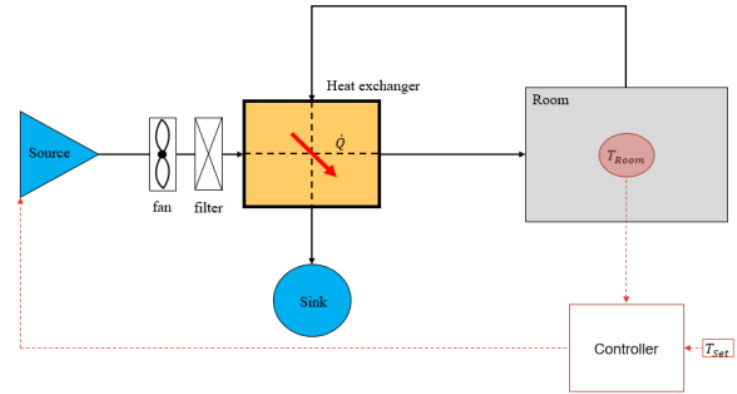

Figure 2: ERV system in Modelica consisting of a ventilator, cross flow heat exchanger, filter, heater, cooler and a room model.

The core component of the ventilation system is the heat exchanger. This is designed as a cross-flow heat exchanger. To create the simulation model for the ventilation system, a heat transfer model is integrated for cross-flow design. According to the Association of German Engineers (VDI), the following equation applies to the effectiveness of heat transfer in cross-flow heat exchanger with fluid mixed on both sides (VDI, 2013):

$$
\varepsilon_{\text {Cross }}=\frac{1}{\frac{1}{1-e^{-N T U}+\frac{R}{1-e^{-R * N T U}}-\frac{1}{N T U}}}
$$

This effectiveness calculation $\varepsilon_{\text {Cross }}$ is implemented as a function in Modelica using the number of transfer units $(N T U)$ and heat capacity flow $R$. In addition, the manufacturer's instructions state that the heat-transferring surface is made of paper. In this respect, a new record is implemented for paper, which converts the material properties into Modelica.

An existing room from the Modelica Buildings model library is used as the room model (Wetter, 2019). This model offers various possibilities for parameterization. These include: geometric dimensions of the room wall, floor and ceiling materials external and internal heat gains port for reading in weather data. The current room temperature is implemented by means of an energy balance over the room.

The filter is modelled as pressure drop $\Delta p$ (in $\mathrm{Pa}$ ) between inlet and outlet of the filter. Assuming particles to be in the air, the filter is getting defiled over time $t$ by dust feed. Hence, it pressure drop increases time-dependently described as follows (Trox, 2012)

$$
\Delta p=6 * 10^{-14} * t^{2}-4 * 10^{-7} * t+70 .
$$

For the other components simple energy and mass balances are introduced to describe the physical behaviour of the system.

Regarding the calibration of the system, five ventilation stages are used. In order to meet these target values for all five ventilation stages and the associated volume flows, a third-degree polynomial is stored, which takes into account the volume flow dependence of the pressure losses in the model. This results in an acceptable calibration quality of $0.861 \mathrm{~W}$ based on a determined RMSE. Table 1 summarises the associated electrical performances. 
Table 1: Calibration of electric output power.

\begin{tabular}{|c|c|c|c|}
\hline Stage & $\begin{array}{c}\boldsymbol{P}_{\text {el,sim }} \\
{[\mathbf{W}]}\end{array}$ & $\begin{array}{c}\boldsymbol{P}_{\text {el,meas }} \\
{[\mathbf{W}]}\end{array}$ & $\begin{array}{c}\boldsymbol{\Delta} \boldsymbol{P}_{\text {el }} \\
{[\mathbf{W}]}\end{array}$ \\
\hline $\mathbf{1}$ & 163 & 165 & -2 \\
\hline $\mathbf{2}$ & 91 & 90 & 1 \\
\hline $\mathbf{3}$ & 42 & 41 & 1 \\
\hline $\mathbf{4}$ & 21 & 22 & 1 \\
\hline $\mathbf{5}$ & 15 & 14 & 1 \\
\hline
\end{tabular}

In the second step, the effectiveness of the heat transfer is calibrated against the measured values given for the five ventilation stages. As before, the generated simulation results are compared with the manufacturer data. It can be stated that the effectiveness of the simulation is underestimated. This is due to the calculation of (VDI, 2013). Real efficiencies are therefore better than the calculated ones. For the model, the effectiveness must be corrected upwards in the course of calibration. Based on the individual deviations between simulated and measured efficiencies, a mean deviation is determined, with which the correction of the calculation is made in the model. This allows a quality of 0.021 to be achieved on the basis of the RMSE. Table 2 shows the associated efficiencies.

Table 2: Calibration of effectiveness.

\begin{tabular}{|c|c|c|c|}
\hline Stage & $\boldsymbol{\varepsilon}_{\text {Cross, sim }}$ & $\boldsymbol{\varepsilon}_{\text {Cross,meas }}$ & $\boldsymbol{\Delta} \boldsymbol{\varepsilon}_{\text {Cross }}$ \\
\hline $\mathbf{1}$ & 0.921 & 0.9 & 0.021 \\
\hline $\mathbf{2}$ & 0.904 & 0.880 & 0.024 \\
\hline $\mathbf{3}$ & 0.858 & 0.855 & 0.003 \\
\hline $\mathbf{4}$ & 0.797 & 0.815 & -0.018 \\
\hline $\mathbf{5}$ & 0.746 & 0.775 & -0.029 \\
\hline
\end{tabular}

In all cases, we have a well modelled and calibrated Digital Twin Instance that can be studied regarding predictive maintenance.

\section{Predictive Maintenance of Filter systems}

As shown in Figure 2, the ventilation system has a filter to cut off particles from the air that is delivered in the room. During operation, the filter is filled with particles. The pressure drop increases. To ensure the adjusted mass flow, the ventilation systems' power consumption increases.

A typical maintenance action would be to exchanger the filter. Using DTD we study different schedules to find the best time for an exchange. During operation, we consider four different scenarios for annual simulations. Herein, different criterions must be met for an exchange:

1) Exchange every year (Baseline scenario)

2) Exchange at $150 \mathrm{~Pa}$ pressure drop

3) Exchange at $200 \mathrm{~Pa}$ pressure drop

4) Exchange every 2.5 years

Assuming a price for electrical power of $29.5 \mathrm{ct} / \mathrm{kWh}$ and $55 €$ per exchanger, we calculate for a lifecycle of ten years total operation costs and energy consumption. Referred to the baseline scenario, the results are shown in Figure 3. (Farr, 2006; Statistische Bundesamt, 2018)

Exchanging the filter once a year, the lowest electric consumption is archived with $3827 \mathrm{kWh}$. The total costs are $1129 €$.
However we calculated the lowest electric energy consumption in the baseline scenario, it is not the scenario with lowest total costs. Scenario 2 and 3 show lower total costs by about 5 to $6 \%$ savings. Simultaneously, the energy consumption increases by about 6 to $10 \%$. Both the highest electric consumption as well as the highest total costs is archived with scenario 4 . Here, total costs increase by about $1 \%$ and electric energy consumption increases about $30 \%$.

- Total costs $[€]$ Energy consumption [kWh]

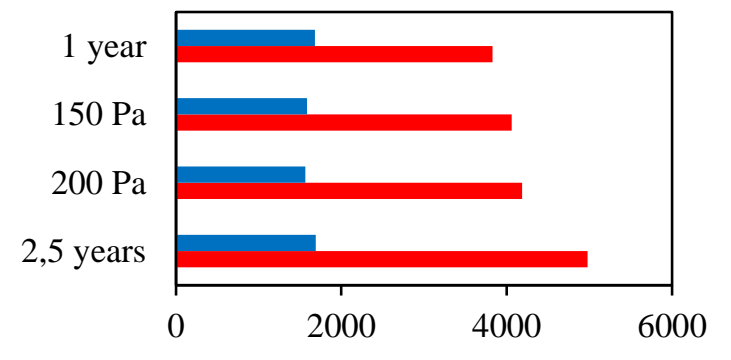

Figure 3: Total costs and electric energy consumption of four different Digital Twin Aggregates.

These fictive scenarios indicate the potential of DTD. If we can provide the digital twin with measurement data and apply fault detection, filter exchanges can be predicted and both total costs and electric consumption can be decreased significantly.

Using these results, Digital Twin Design shows potential for the whole systems' lifecycle efficiency. Applying this to other, more sophisticated control and maintenance scenarios higher savings can be reached, respectively. Hence, we showed that DTD, consisting of PLM and digital twins, was successfully applied to HVAV systems and the lifecycle efficiency could be increased due to predictive maintenance.

\section{Conclusion}

This work shows that Digital Twin Design can be applied to HVAC systems using Product Lifecycle Management and digital twins for life cycle analysis. On the basis of five life cycle phases, Digital Twin Concept, Digital Twin Prototype, Digital Twin Instance, Digital Twin Aggregate and Digital Twin Knowledge, the presented approach serves as a detailed framework for the implementation of a digital twin to a whole product lifecycle.

To demonstrate the feasibility of the developed approach, a grey box model of a ventilation system was created and manually calibrated. In addition, a communication structure based on MQTT was presented.

The potential of predictive maintenance with various routines with regard to air filter replacement for the ventilation system was successfully demonstrated. These are based on the existence of an optimum of energy consumption and total costs. Savings of total costs up to $10 \%$ are predicted using filter exchanges at a pressure loss of $200 \mathrm{~Pa}$. By integrating a digital twin for HVAC systems, it is therefore possible to increase lifecycle efficiency in terms of both energy and total costs. 
Due to the complexity and interdisciplinary of the topic of digital twin, not all phases of the DTD approach could be tested for applicability and practicability in this work. Consequently, there is a need for continued research work, particularly in the areas of machine communication and software technology.

Overall, the full potential of digital twins is not exhausted, yet. Rather, the technology is still in its infancy. In the future, further developments in and with digital twins are to be expected. Their use is highly likely to multiply in the coming years, as the basic infrastructure for implementation is already in place.

In addition, some interesting approaches and ideas for the use of digital twins have already emerged. Only the implementation in practice is still open in many cases. It will also be exciting to observe the extent to which new business models with digital twins will occur. The market for applications with digital twins is, in any case, almost endlessly large in all sectors as digitalization progresses.

\section{References}

Afram, A., Janabi-Sharifi, F. (2014). Theory and applications of HVAC control systems - A review of model predictive control (MPC). In: Building and Environment 72, 343-355.

Dongellini, M. et al. (2017). Sizing effects on the energy performance of reversible air-source heat pumps for office buildings. In: Applied Thermal Engineering, 1073-1081.

Poppi, S. et al. (2016). Influence of boundary conditions and component size on electricity demand in solar thermal and heat pump combisystems. In: Applied Energy 162, 1062-1073.

Stark, J. (2015). Product Lifecycle Management. Cham : Springer International Publishing, (Decision Engineering).

Staffel, I. et al. (2012). A review of domestic heat pumps. In: Energy Environ. Sci. (5), 9291-9306.

Arbeitsgemeinschaft Energiebilanzen e.V. (2017). Auswertungstabellen zur Energiebilanz Deutschland: $1990 \quad$ bis 2016, http://www.agenergiebilanzen.de/index.php?article_id=29\&fileNa me=ausw_24juli2017_ov.pdf.

Tao, F. et al. (2018). Digital twin-driven product design framework. In: International Journal of Production Research 12 (1), 1-19.

Yoga Mule, J. (2012). Concept and Evolution of PLM. In: International Journal of Applied Information Systems (4), 25-28.

Schleich, B. et al. (2017). Shaping the digital twin for design and production engineering. In: CIRP Annals 66 (1), 141-144.
Tuegel, E.J. et al. (2011). Reengineering Aircraft Structural Life Prediction Using a Digital Twin. In: International Journal of Aerospace Engineering (3), $1-14$.

Grieves, M. (2014). Digital Twin:Manufacturing Excellence through Virtual Factory Replication.

Mueller, D. (2016). Simulationsmodelle für die Heiz- und Raumlufttechnik: Heizflächen. Lecture, RWTH Aachen University, Aachen.

Li, C. et al. (2017). A dynamic Bayesian network approach for digital twin. In: 19th AIAA NonDeterministic Approaches Conference.

Chong, A., Menberg, K. (2018). Guidelines for the Bayesian calibration of building energy models. In: Energy and Buildings 174, 527-547.

Mehdiyev, N. et al. (2016). Evaluating Forecasting Methods by Considering Different Accuracy Measures. In: Procedia Computer Science 95, 264-271.

HIVEMQ ENTERPRISE MQTT BROKER (2019). MQTT 101 - How to Get Started with the lightweight IoT Protocol, https://www.hivemq.com/blog/how-toget-started-with-mqtt.

Hirmer, P. et al. (2016). Automating the Provisioning and Configuration of Devices in the Internet of Things. In: Complex Systems Informatics and Modeling Quarterly (9), 28-43.

Haag, S., Anderl, R. (2018). Digital twin - Proof of concept. In: Manufacturing Letters 15, 64-66.

Bauer, R. (2018). What's the Diff: VMs vs Containers, https://www.backblaze.com/blog/vm-vs-containers/.

Schreiner, J., Mossner, J. (2018). IoT-Basics: Was ist Predictive Maintenance?, https://www.industry-ofthings.de/iot-basics-was-ist-predictive-maintenancea-693842/.

Meier, A. (2018). Werkzeuge der digitalen Wirtschaft: Big Data, NoSQL \& Co. Wiesbaden: Springer Fachmedien Wiesbaden.

Verein Deutscher Ingenieure (2013). VDI-Wärmeatlas: Mit 320 Tabellen. 11. Aufl. //11. bearb. und erw. Aufl. Berlin: Springer Berlin and Springer Vieweg.

Wetter, M. (2019). Modelica Buildings Library. http://simulationresearch.lbl.gov/modelica/.

Trox Technik (2012). Filtertechnologie, https://www.chillventa.de/Filestore.aspx/F08_P2_TR OX.

Farr, Camfil (2006). Saubere Luft mit geringen Kosten, http://www.camfil.de.

Statistische Bundesamt (Destatis) (2018). Daten zur Energiepreisentwicklung - Lange Reihen von Januar 2005 bis Oktober 2018. 